8-1-2008

\title{
Mixing in Shallow Cumulus Clouds Studied by Lagrangian Particle Tracking
}

Thijs Heus

Cleveland State University, t.heus@csuohio.edu

Gertjan van Dijk

Delft University of Technology

Harmen J.J. Jonker

Delft University of Technology, h.j.j.jonker@tudelft.nl

Harry E.A. Van den Akker

Delft University of Technology

Follow this and additional works at: https://engagedscholarship.csuohio.edu/sciphysics_facpub

Part of the Physics Commons

How does access to this work benefit you? Let us know!

Publisher's Statement

(C) Copyright 2008 AMS

\section{Repository Citation}

Heus, Thijs; van Dijk, Gertjan; Jonker, Harmen J.J.; and Van den Akker, Harry E.A., "Mixing in Shallow Cumulus Clouds Studied by Lagrangian Particle Tracking" (2008). Physics Faculty Publications. 222.

https://engagedscholarship.csuohio.edu/sciphysics_facpub/222

This Article is brought to you for free and open access by the Physics Department at EngagedScholarship@CSU. It has been accepted for inclusion in Physics Faculty Publications by an authorized administrator of

EngagedScholarship@CSU. For more information, please contact library.es@csuohio.edu. 


\title{
Mixing in Shallow Cumulus Clouds Studied by Lagrangian Particle Tracking
}

\author{
Thijs Heus, Gertuan van Dijk, Harm J. J. Jonker, and Harry E. A. Van den Akker \\ Department of Multi-Scale Physics, Delft University of Technology, Delft, Netherlands
}

(Manuscript received 24 July 2007, in final form 15 November 2007)

\begin{abstract}
Mixing between shallow cumulus clouds and their environment is studied using large-eddy simulations. The origin of in-cloud air is studied by two distinct methods: 1) by analyzing conserved variable mixing diagrams (Paluch diagrams) and 2) by tracing back cloud-air parcels represented by massless Lagrangian particles that follow the flow. The obtained Paluch diagrams are found to be similar to many results in the literature, but the source of entrained air found by particle tracking deviates from the source inferred from the Paluch analysis. Whereas the classical Paluch analysis seems to provide some evidence for cloud-top mixing, particle tracking shows that virtually all mixing occurs laterally. Particle trajectories averaged over the entire cloud ensemble also clearly indicate the absence of significant cloud-top mixing in shallow cumulus clouds.
\end{abstract}

\section{Introduction}

The interaction between cumulus clouds and their environment has been a much debated issue for several decades. Stommel (1947) based his cloud model on the concept of a laterally entraining plume, but Squires (1958) argued that cloud-top mixing and the resulting penetrative downdrafts are better able to predict the behavior of cumuli. In this way, he could, for instance, better explain why liquid water content tends to be relatively constant throughout lateral transects. Later measurements found a lack of mean horizontal flow into the cloud (e.g., Telford and Wagner 1974) in support of this view. On the other hand, Heymsfield et al. (1978) found significant lateral mixing in a sheared environment for the nonbuoyant parts of the cloud. This diminishes the size of the moist adiabatic cloud core but leaves the rest of the core largely undiluted.

In the following years, the discussion on cloudenvironment interaction was dominated by the use of conserved variable diagrams, as introduced in this field by the key paper of Paluch (1979, hereafter P79). She plotted the phase space of two conserved variables (equivalent potential temperature $\theta_{q}$ and the total water content $q_{t}$ ) of in-cloud air. These in-cloud proper-

Corresponding author address: Thijs Heus, Dept. of Multi-Scale Physics, Delft University of Technology, P.O. Box 5046, 2600 GA Delft, Netherlands.

E-mail: t.heus@tudelft.nl ties, then, have to be a linear combination of the environmental values at the heights from where the entrained air originates. Because P79 found the in-cloud properties for large rising cumuli to lie on a line between cloud base and cloud top, she concluded it highly likely that the source level of entrained air lies at cloud top.

More recent observations (e.g., Betts 1982; Jensen et al. 1985; LaMontagne and Telford 1983; Reuter and Yau 1987b) obtained similar results for different pairs of conserved variables, especially in the upper part of the cloud. Others, such as Raymond and Wilkening (1982), Blyth et al. (1988), Taylor and Baker (1991; hereafter TB91), Neggers et al. (2002), and Zhao and Austin (2005a), observed source levels close to observation level, either in observations or in large-eddy simulations (LES). These sources around observation level, in particular, are found for the lower and middle parts of the cloud and are usually explained by a buoyancy-sorting mechanism. In such a mechanism, a cloud core is defined as being positively buoyant with regard to the environment $\left(\theta_{v, \text { core }}>\theta_{v \text {,env }}\right)$. It contains parcels of cloudy air rising to their level of neutral buoyancy, where they mix with the environment, evaporate, and are eliminated from the ensemble. TB91 showed that in-cloud observations might appear to be linear combinations of air from two sources on a Paluch diagram but can also be explained by mixing from multiple sources followed by buoyancy sorting. Such a mechanism yields a triangular area that is usually pointing in the direction 
of observation level in the Paluch diagram, meaning that in-cloud parcels are distributed along a line. This lateral mixing also forms the basis for the mass-flux approach in many operational parameterizations (e.g., Kain and Fritsch 1993; Siebesma and Cuijpers 1995; von Salzen and McFarlane 2002).

Blyth (1993) emphasized the role of a recirculating vortex at the top of the thermal in cloud mixing. This recirculation can be associated with a descending shell of air around the cloud (e.g., Reuter and Yau 1987a; Jonas 1990). Rodts et al. (2003) and Heus and Jonker (2008) found that this descending shell is also observed at lower heights and is due to evaporative cooling induced by lateral mixing. This shell is capable of dragging significant amounts of air downward alongside the cloud, thus potentially explaining the observation of source levels in Paluch diagrams above observation levels in situations of dominant lateral mixing.

With the advance of radar measurements and the increase of computer power in the last decade, it became possible to attain more direct evidence for cloudenvironment interaction. Damiani et al. (2006) drew streamlines through radar observations, and others such as Lin and Arakawa (1997) and Carpenter et al. (1998) calculated backward trajectories through numerical simulations. Dispersion of a passive scalar allowed Zhao and Austin (2005b) to improve understanding of the role of the recirculating vortex on top of the ascending cloud top. The mechanisms and importance of mixing with the bulk of the cloud, however, remain unclear.

This study is an attempt to resolve the issue of cloudenvironment mixing of shallow cumuli by departing from the implicit Paluch analysis and determining the origin of in-cloud air explicitly. This is done by incorporating into LES many massless tracer particles that follow the flow and thus can represent in-cloud air parcels from a Lagrangian perspective. Such an approach has been followed before by Weil et al. (2004) and Dosio et al. (2005), for example, for the clear convective boundary layer. Because tracer particles are uniquely identifiable and follow the motion of air, they can serve as a powerful means to study the history of an individual parcel of in-cloud air. This way, the origin of the air inside a cloud can be found without imposing strong assumptions. The advantage of LES is that the cloud and its flow, temperature, and moisture fields are known completely, with temporal and spatial resolutions that are difficult to achieve in observational campaigns. LES has been widely used in various contexts and has been extensively validated for studies of the dynamics of nonprecipitating cumulus clouds (e.g., Heus and Jonker 2007; Siebesma and Jonker 2000;
Siebesma et al. 2003). Thus, LES is capable of simulating a cloud field consisting of a large number of independent clouds, and a solid statistical approach can be made.

The first part of this paper (section 3) consists of a comparison between the results using conserved variable diagrams like P79 and results obtained from particle tracking. The purpose of this exercise is twofold: 1 ) to see whether LES can obtain results similar to observations and, if so, 2) to study the origin of the particles and compare it to the origin of the in-cloud air as inferred from the Paluch analysis. This analysis is performed by investigating a number of individual clouds, chosen to be similar to many observed clouds described in the literature.

The second part of this work treats cloud-environment interaction from a broader point of view. The focus shifts toward the ensemble average of the cloud field, instead of measurements of individual clouds that can easily be subject to stochastic events. By careful normalization and conditional sampling of clouds, the focus remains on the behavior of the individual but average cloud.

Discussion of the average motion of the particles in this fashion enables one to study the role of various entrainment and detrainment models:

- cloud-top entrainment followed by penetrative downdrafts (Squires 1958);

- entrainment and detrainment induced by a recirculating vortex at cloud top (Blyth et al. 1988);

- and entrainment and detrainment at the side of the cloud, either in the classical view of the shedding thermal (Stommel 1947), buoyancy-sorting (TB91), or with some additional downward motion due to the subsiding shell (Heus and Jonker 2007).

Finally, the results of the previous sections are discussed in relation with the role of clouds in transport through the CBL.

\section{Case setup}

The simulations were run with the parallelized Dutch atmospheric LES (DALES) model as described by Cuijpers and Duynkerke (1993). The numerical case used in the study is based on the Barbados Oceanographic and Meteorological Experiment (BOMEX; Holland and Rasmusson 1973). Because this marine cumulus case has no diurnal cycle and is almost in steady state, long simulations can be performed and the cloud field can be considered statistically stationary over the entire run. Sensible and latent surface heat fluxes amount to 8 and $150 \mathrm{~W} \mathrm{~m}^{-2}$, respectively (resulting in 
a Bowen ratio of $\left.r_{B}=0.05\right)$. Cloud base is located around $500 \mathrm{~m}$, and the inversion layer lies between 1500 and $2000 \mathrm{~m}$; the typical horizontal cloud size is around $500 \mathrm{~m}$. BOMEX shows a relatively large mean vertical shear, up to $1.8 \mathrm{~m} \mathrm{~s}^{-1} \mathrm{~km}^{-1}$. More detailed information on the numerical case can be found in the intercomparison study by Siebesma et al. (2003).

Simulations were carried out on a domain of $6.4 \mathrm{~km} \times$ $6.4 \mathrm{~km} \times 3.2 \mathrm{~km}$, with a resolution of $\Delta x=\Delta y=25 \mathrm{~m}$, $\Delta z=20 \mathrm{~m}$ and a time step of $\Delta t=1 \mathrm{~s}$. Three simulations (statistically identical but each with a different random perturbation of the initial field) with a duration of $8 \mathrm{~h}$ each were performed; the first $3 \mathrm{~h}$ were discarded as spinup.

The Lagrangian Particle Dispersion Model (LPDM) was based on the work of Thomson (1987) and on the implementation of the model in LES by Weil et al. (2004). Here, the equation of motion for the tracer particles is

$$
\frac{d \mathbf{x}}{d t}=\tilde{\mathbf{u}}(\mathbf{x}, t)+\mathbf{u}^{\prime}(\mathbf{x}, t),
$$

where $\tilde{\mathbf{u}}$ is the LES-resolved velocity and $\mathbf{u}^{\prime}$ is a contribution to the particle velocity from subfilter-scale (SFS) fluctuations; $\mathbf{u}^{\prime}$ is modeled as a Gaussian random term the magnitude of which is determined by the SFS turbulent kinetic energy (TKE) of the Eulerian LES field. Resolved velocities and conserved properties (e.g., the liquid potential temperature $\theta_{l}$ and the total water content $q_{t}$ ) are interpolated linearly to the position of the particle; other scalars, such as the liquid water content $q_{l}$ and the virtual potential temperature $\theta_{v}$, are calculated using the interpolated conserved variables. For time integration, the second-order Adams-Bashforth method is used. Boundary conditions are kept the same as in the LES, meaning no-slip conditions at top and bottom and periodic boundary conditions in the horizontal directions. In all simulations, $128 \times 128 \times 80=$ $1.3 \times 10^{6}$ particles are distributed homogeneously throughout the domain. A detailed validation of the particle model is provided in the appendix. As is shown there, the Lagrangian particles follow the flow within the bulk of the CBL very well for a simulation of BOMEX.

\section{Detailed cloud investigation}

\section{a. Terminology}

The first approach to analyze cloud-environment interaction consists of looking into individual clouds and comparing the results of Paluch diagrams with results from particle tracking. For each of the three simulations, at observation times $t_{\mathrm{obs}}=4 \mathrm{~h}, t_{\mathrm{obs}}=5 \mathrm{~h}$, and $t_{\mathrm{obs}}=6 \mathrm{~h}$, the tallest cloud is selected from the cloud field. This approach is not only followed because of the importance of large clouds in the transport of air through the CBL but also because the vast majority of previous work focuses on large active clouds, allowing for a good comparison. Because these nine clouds yielded similar results, only one of them is discussed in detail here. This cloud has a cloud base at $550 \mathrm{~m}$ and a cloud top at $1750 \mathrm{~m}$ (which is in the middle of the inversion layer).

The construction of a Paluch diagram allows freedom in choosing a conserved variable pair. Traditionally, the total humidity $q_{t}$ is chosen as one variable and is put on the $y$ axis of the diagram. Because the $y$ axis is easily associated with height, it is useful to plot the diagram with a downward-pointing $q_{t}$ axis.

The $x$ axis is traditionally used for a temperature scale. Which conserved temperature scale is used varies from study to study; P79 used the equivalent potential temperature $\theta_{q}$, while others used, for instance, the liquid potential temperature $\theta_{l}$. In the simplest approximation, $\theta_{q}$ and $\theta_{l}$ are related to each other by

$$
\theta_{q} \approx \theta_{l}+\frac{L}{c_{p}} q_{t}
$$

where $L=2.5 \times 10^{6} \mathrm{~J} \mathrm{~kg}^{-1}$ is the latent heat of vaporization and $c_{p}=1004 \mathrm{~J} \mathrm{~kg}^{-1} \mathrm{~K}^{-1}$ is the specific heat of dry air. Note that because $\theta_{l}$ and $q_{t}$ are conserved, so too is the approximated $\theta_{q}$. The importance of an appropriate temperature scale is illustrated in Fig. 1. In the two top panels, the outline of a Paluch diagram is shown with $\left(\theta_{l}, q_{t}\right)$ and $\left(\theta_{q}, q_{t}\right)$ as conserved variable pairs. The area enclosed by the environmental curve and a dashed line drawn between cloud-base $\mathrm{CB}_{\text {env }}$ and cloud-top $\mathrm{CT}_{\text {env }}$ is where the properties of in-cloud air mostly lie. Within such an elongated shape, any uncertainty in either the measurements or the linear fit would clearly yield large uncertainties in the intersecting points near cloud-base $z_{i b}$ and near cloud-top $z_{i t}$. However, any linear combination of $\theta_{l}$ and $\theta_{q}$ is also a conserved variable itself. Therefore, by defining an alternative temperature scale $\theta_{\alpha}$ as

$$
\theta_{\alpha}=(1-\alpha) \theta_{l}+\alpha \theta_{q}
$$

and maximizing the area between the environmental curve and the $\mathrm{CT}_{\text {env }}-\mathrm{CB}_{\text {env }}$ line as a function of $\alpha$, an optimal temperature scale $\theta_{\alpha}$ can be found, as illustrated in the third panel of Fig. 1. For this particular case, $\alpha=0.272$ was found. It should be stressed that the choice of $\alpha$ is arbitrary in that we do not attribute any direct physical meaning to $\theta_{\alpha}$, but for the fact that it is a conserved variable in the same sense that $\theta_{l}$ and $\theta_{q}$ are. So that we are still allowed to construct a Paluch 

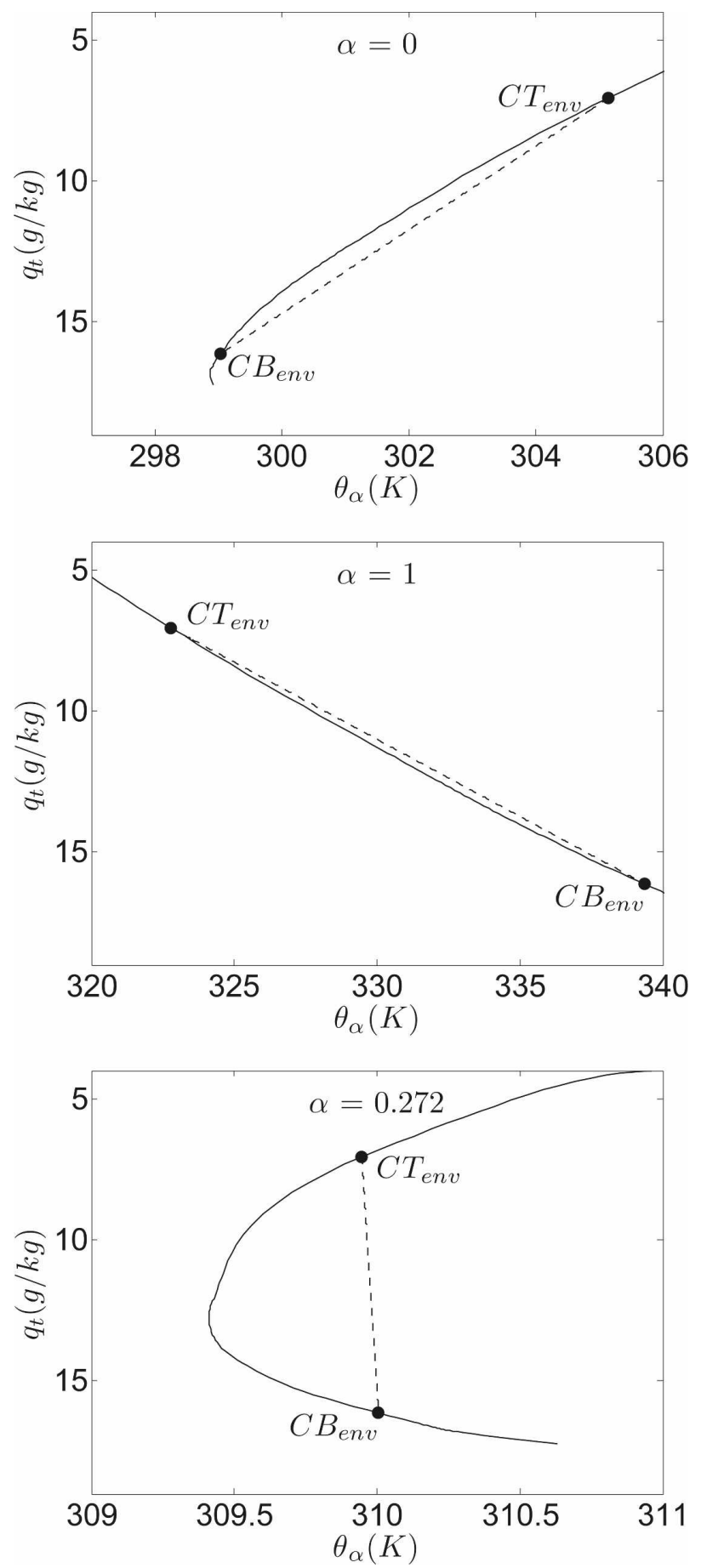

FIG. 1. Paluch diagram for several pairs of conserved variables $\left(\theta_{\alpha}\right.$, $q_{t}$ ) with $\theta_{\alpha}=(1-\alpha) \theta_{l}+\alpha \theta_{q}$. (top to bottom) Here, $\alpha=0\left(\theta_{\alpha}=\theta_{l}\right)$, $\alpha=1\left(\theta_{\alpha}=\theta_{q}\right)$ and $\theta_{\alpha}$ for $\alpha=0.272$, where the area between the environmental curve and the line between $\mathrm{CB}_{\text {env }}$ and $\mathrm{CT}_{\text {env }}$ is at a maximum. diagram with it, any conclusion that holds in $\left(\theta_{\alpha}, q_{t}\right)$ space should also hold in $\left(\theta_{l}, q_{t}\right)$ space. The only reason to introduce $\theta_{\alpha}$ is that the graphical inspection on which the upcoming discussion relies is virtually impossible with either $\theta_{l}$ or $\theta_{q}$ as a temperature scale, as is illustrated in Fig. 1.

Because Paluch diagrams are representations of the physical world in a more abstract phase space, these diagrams can easily become rather complicated. A further complication here is the tracking of air parcels in time. In Fig. 2, four fictitious air parcels are shown, both in a cloud and in the corresponding Paluch diagram. These parcels are observed within the cloud at $t^{\prime} \equiv t-t_{\mathrm{obs}}=0$ at height $z_{\mathrm{obs}}$ and are denoted as open circles in the figures. In the Paluch diagram, the environment is shown as a solid line, with squares at 200-m height intervals. Four gray crosses at the environmental curve denote (from bottom to top along the environmental curve) the in-cloud properties of cloud-base $\mathrm{CB}_{\text {cld }}$ (representing the properties of the inflow from the subcloud layer), the height of cloud-base $\mathrm{CB}_{\text {env }}$, the observation height $z_{\text {obs }}$, and cloud-top $\mathrm{CT}_{\text {env }}$. The dashed line representing the best fit in phase space through the parcels at $t_{\text {obs }}$ crosses the environmental curve at $z_{i t}$ and $z_{i b}$. The location of $z_{i b}$ can be interpreted as the average of the lower part of sources. If the presence of subcloud-layer air is dominating the sampling, $z_{i b}$ lies near $\mathrm{CB}_{\text {cld }}$. Similarly, $z_{i t}$ represents the upper part of the sources. If cloud mixing is modeled as mixing between two sources, these sources lie at $z_{i t}$ and $z_{i b}$. The source at $z_{i b}$ is then associated with inflow from the subcloud layer, and $z_{i t}$ with the source of entrained air. This is the main assumption underlying the analysis of the Paluch diagram, and it is usually justified by the high correlation between the linear fit and the properties of the air parcels. As TB91 showed, this only holds if either buoyancy sorting does not apply or if the regions allowed by buoyancy sorting are sufficiently large.

Because the particles in the LPDM representing the air parcels can be tracked backward in time, the position of the parcels before observation time $t^{\prime}=0$ can now also be plotted in the Paluch diagram. For some time $t^{\prime}<0$, the parcels are shown as solid dots. Before entering the cloud, the fictitious parcels in Fig. 2 (of which one originates from the subcloud layer, two from around observation level, and one from above cloud top) have the same properties as the environment at their respective heights. The aim of this paper is to study the origins of air entrained from the cloud layer into the cloud. Thus, in the calculation of the average height of the parcels, the subcloud layer must be excluded. This average height of parcels that reside above 



FIG. 2. (top) Conceptual picture of air parcels moving through a cloud and (bottom) through the corresponding Paluch diagram. Crosses on the environmental curve denote (top to bottom) cloud top $\left(\mathrm{CT}_{\text {env }}\right)$, observation height $\left(z_{\text {obs }}\right)$, cloud base $\left(\mathrm{CB}_{\text {env }}\right)$, and the average in-cloud cloud-base properties $\mathrm{CB}_{\text {cld }}$. This is the average of all air parcels in the cloud at $t=t_{\mathrm{obs}}$ and $z=z_{\mathrm{cb}}$. Squares denote intervals of $200 \mathrm{~m}$. Circles signify parcels in the cloud at $t=t_{\mathrm{obs}}$ and $z=z_{\mathrm{obs}}$. The dashed line is a best linear fit through the parcels at $t=t_{\mathrm{obs}}$ and $z=z_{\mathrm{obs}}$, and it crosses the environmental curve at $z=z_{i b}$ and at $z=z_{i t}$. The average height of the parcels residing in the cloud layer is marked with the large dot labeled $\langle z\rangle_{p}$.

cloud base is indicated by a large gray dot on the environmental curve and denoted by $\langle z\rangle_{p}\left(t^{\prime}\right)$.

For quick reference, an overview of the definitions used in this section is given in Table 1 .
TABLE 1 . Summary of the key variables used in section 3 .

\begin{tabular}{ll}
\hline \hline Variable & \multicolumn{1}{c}{ Description } \\
\hline$\theta_{\alpha}$ & $(1-\alpha) \theta_{l}+\alpha \theta_{q} ;$ optimized temperature scale \\
$\alpha$ & $0.272 ;$ optimization parameter in $\theta_{\alpha}$ \\
$\mathrm{CB}_{\text {env }}$ & Environmental properties at cloud-base height \\
$\mathrm{CB}_{\mathrm{cld}}$ & In-cloud properties at cloud-base height \\
$\mathrm{CT}_{\text {env }}$ & Environmental properties at cloud-top height \\
$t_{\mathrm{obs}}$ & Moment of observation \\
$t^{\prime}$ & $t-\mathrm{t}_{\text {obs}} ;$ time relative to observation time \\
$z_{\mathrm{obs}}$ & Height of observation \\
$z_{i b}$ & Lower source level inferred from Paluch diagram \\
$z_{i t}$ & Upper source level inferred from Paluch diagram \\
$\langle z\rangle_{p}\left(t^{\prime}\right)$ & Average height of air parcels residing above cloud \\
& base \\
$z_{s}$ & $\langle z\rangle_{p}\left(t^{\prime}=-1800 \mathrm{~s}\right) ;$ average source level of \\
& cloud-layer parcels \\
\hline
\end{tabular}

\section{b. Results and interpretation}

In Fig. 3, Paluch diagrams of the simulations are shown for four observation levels $z_{\text {obs }}$ within the cloud. Additionally, the TB91 buoyancy-sorting regions are enclosed by gray lines denoting the saturation level $q_{s}$, a line between cloud base/observation level, and a gray area where $\theta_{v}<\theta_{v \text {,env }}$. Note that the latter is sharply influenced by the presence of liquid water.

Assuming buoyancy sorting, all points below the $\mathrm{CB}_{\text {cld }} / z_{\text {obs }}$ line come from below and should be positively buoyant; all points above this line come from above and should be negatively buoyant. Note that because of the use of $\theta_{\alpha}$, the allowed regions are (optically) significantly larger than in the $\theta_{q}$ plots originally used in TB91.

In all figures, the vast majority of points comes from below, and they are positively buoyant. However, a few appear to come from above, while from $z_{\mathrm{obs}}=1000 \mathrm{~m}$ upward, the parcels from which almost all of the water has been evaporated are clearly negatively buoyant despite coming from below. This can be explained either by the buoyancy reduction due to evaporation itself or because these parcels overshot their buoyancy-sorting level. This has also been observed by Neggers et al. (2002), for instance. Clearly, for $z_{\mathrm{obs}}=1600 \mathrm{~m}$, the cloud has reached the inversion layer and all parcels are in such an overshoot. This is also the only height where two-point mixing is clearly not valid, because the points are no longer distributed along a line. For all other heights, the points are distributed along a line with a tendency stronger than can be explained by buoyancy sorting alone.

For all heights, the inferred upper-source-level $z_{i t}$ lies around or slightly above observation level. It should be noted that the inferred upper source levels are markedly different from an orthodox cloud-top entrainment 

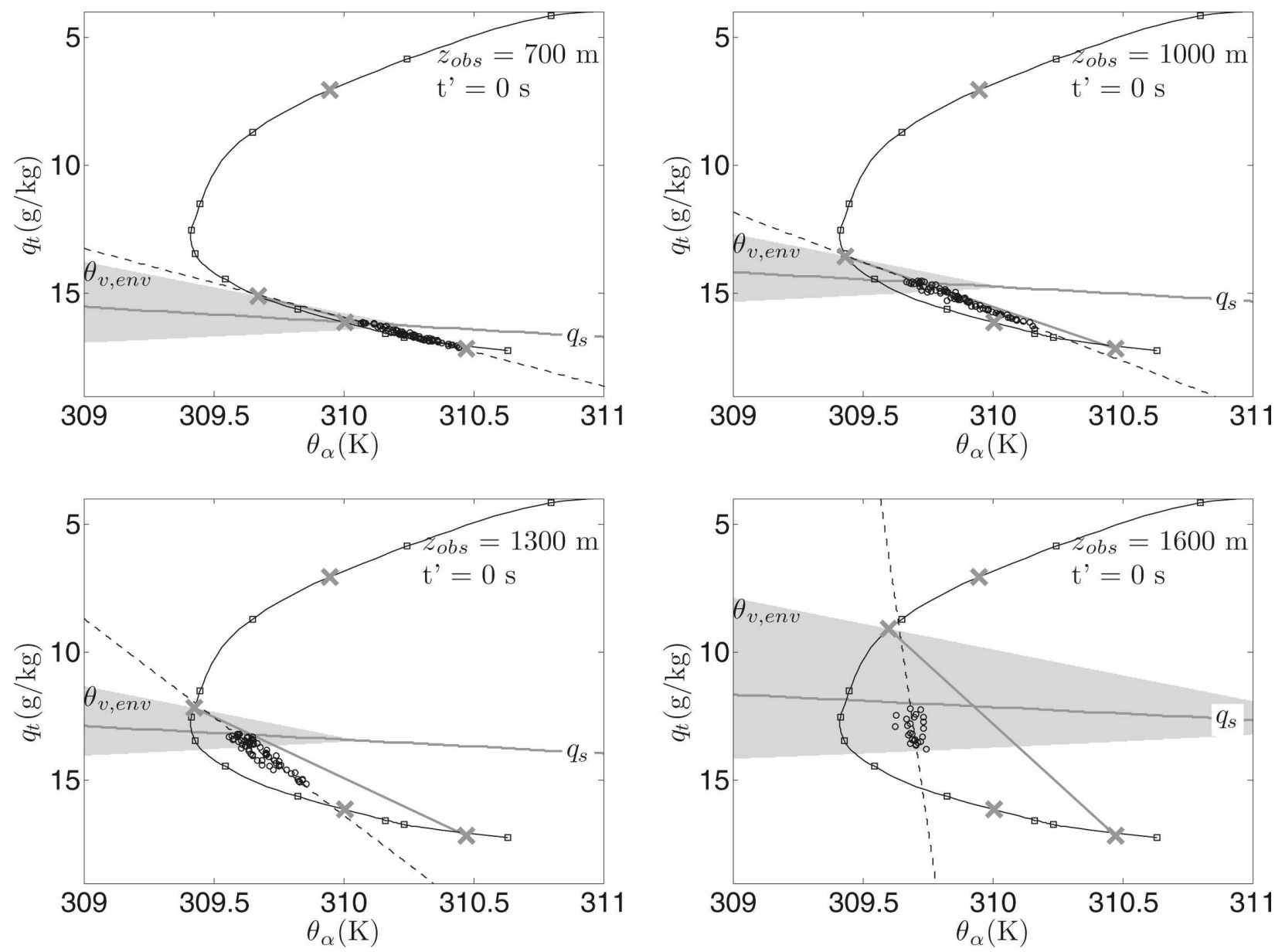

FIG. 3. Paluch diagram at observation level $z_{\mathrm{obs}}=700,1000,1300$, and $1600 \mathrm{~m}$, respectively. The allowed regions of buoyancy sorting of TB91 are enclosed by one gray line that denotes the saturation level $q_{s}$ and another gray line between $\mathrm{CB}_{\mathrm{cld}}$ and $z_{\mathrm{obs}}$. In the shaded area, $\theta_{v}<\theta_{v, \text { env }}$. See Fig. 2 for an explanation of the other symbols.

view, where for all observation heights the uppersource-level $z_{i t}$ should be located at cloud top and the lower-source-level $z_{i b}$ at cloud base. Within the framework of cloud-top entrainment, this is usually explained by air being entrained in an earlier life stage of the cloud when the ascending cloud top of the growing cloud passed the observation level. However, the increase in height of the lower-source-level $z_{i b}$ (reaching $650 \mathrm{~m}$ at $z_{\text {obs }}=1600 \mathrm{~m}$ and only equaling $\mathrm{CB}_{\text {env }}$ in passing at $z_{\mathrm{obs}}=1300 \mathrm{~m}$ ) also hints at lateral entrainment. This increase of $z_{i b}$ has also been observed by LaMontagne and Telford (1983).

Blyth et al. (1988) plotted the source-level $z_{i t}$ as a function of observation-level $z_{\text {obs. }}$. In Fig. 4, their result is shown, accompanied by the same analysis of the current data. Both results display an upper source level that lies at or somewhat above observation level. For higher altitudes, $z_{i t}$ is especially higher than $z_{\text {obs }}$; Blyth (1993) associated this with entrainment induced by the recirculating vortex at cloud top. In any case, the results from simulations of BOMEX look similar to the observations in Blyth et al. (1988) of much deeper cumuli (cloud top up to $6 \mathrm{~km}$ ), regardless of the interpretation of $z_{i t}$.

In Fig. 5, the evolution of particles located at $z_{\text {obs }}=$ $1000 \mathrm{~m}$ at $t^{\prime}=0 \mathrm{~s}$ is shown at previous times. Clearly, most particles present in the cloud layer before $t^{\prime}=$ $-600 \mathrm{~s}$ are concentrated around or below $z_{\mathrm{obs}}$. Indeed, the average level of cloud-layer particles $\langle z\rangle_{p}\left(t^{\prime}=-600\right.$ s) lies below both $z_{\text {obs }}$ and $z_{i t}$ in $\left(q_{t}, \theta_{\alpha}\right)$ phase space. This suggests that at least for this height, the inferred upper-source-level $z_{i t}$ overestimates the true source level of the in-cloud air.

To get a better idea of the location of the particles, the probability density function (PDF) of the height of the particles is shown in Fig. 6. Two processes are clearly visible here. The particles in the subcloud layer (below $550 \mathrm{~m}$ ) are initially homogeneously distributed 



FIG. 4. The upper-source-level $z_{i t}$ as a function of observation height $z_{\text {obs. }}$ (top) Observational results for Montana cumuli from Blyth et al. (1988); (bottom) current LES results of BOMEX.

$\left(t^{\prime}=-3600 \mathrm{~s}\right)$, then start to congregate near the surface $\left(t^{\prime}=-1800 \mathrm{~s}\right)$, and finally rise into the cloud in the 10 min before $t_{\text {obs. }}$ Irrespective of this subcloud process, the PDF in the cloud layer only changes because of the influence of the cloud toward $z_{\text {obs }}$ in the final $10 \mathrm{~min}$ before observation time; going further backward in time, the PDF is broadened because of some turbulent diffusion. This could be expected, because the cloud has a typical lifetime of less than half an hour. Therefore, we define the location of cloud-layer particles $\langle z\rangle_{p}$ at $t^{\prime}=-1800 \mathrm{~s}$ as the source-level $z_{s}$ of the air; the cloud has not yet entrained the particle, and the low cloud fraction of around $10 \%$ makes it highly unlikely that earlier clouds are still influencing the trajectory of the particles. In calculation of $z_{s}$, only the particles that remain above cloud base during the entire period between $t^{\prime}=-1800$ and $t^{\prime}=0 \mathrm{~s}$ have been taken into
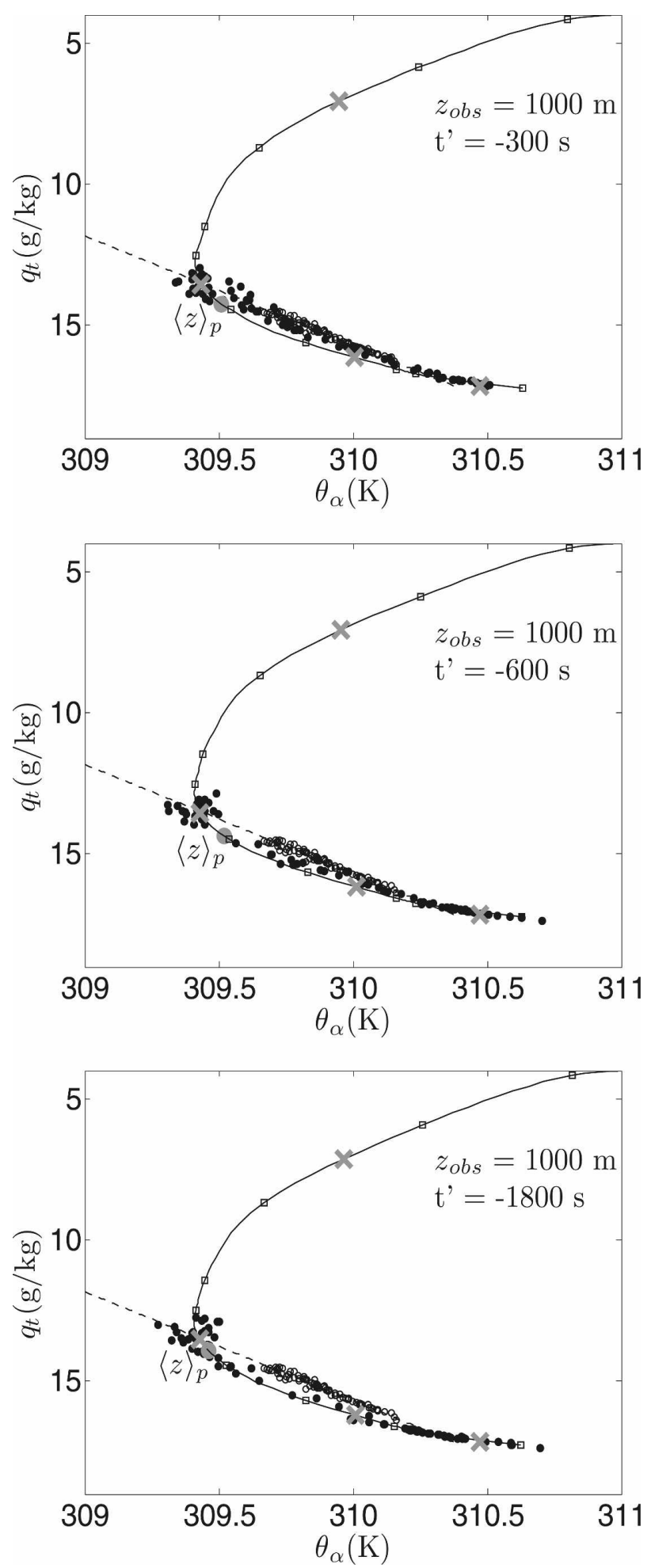

FIG. 5. Paluch diagram evolving in time for $z_{o b s}=1000 \mathrm{~m}$. (top to bottom) Instances where $t^{\prime}=-300, t^{\prime}=-600$, and $t^{\prime}=$ -1800 s. See Fig. 2 for an explanation of the symbols. 

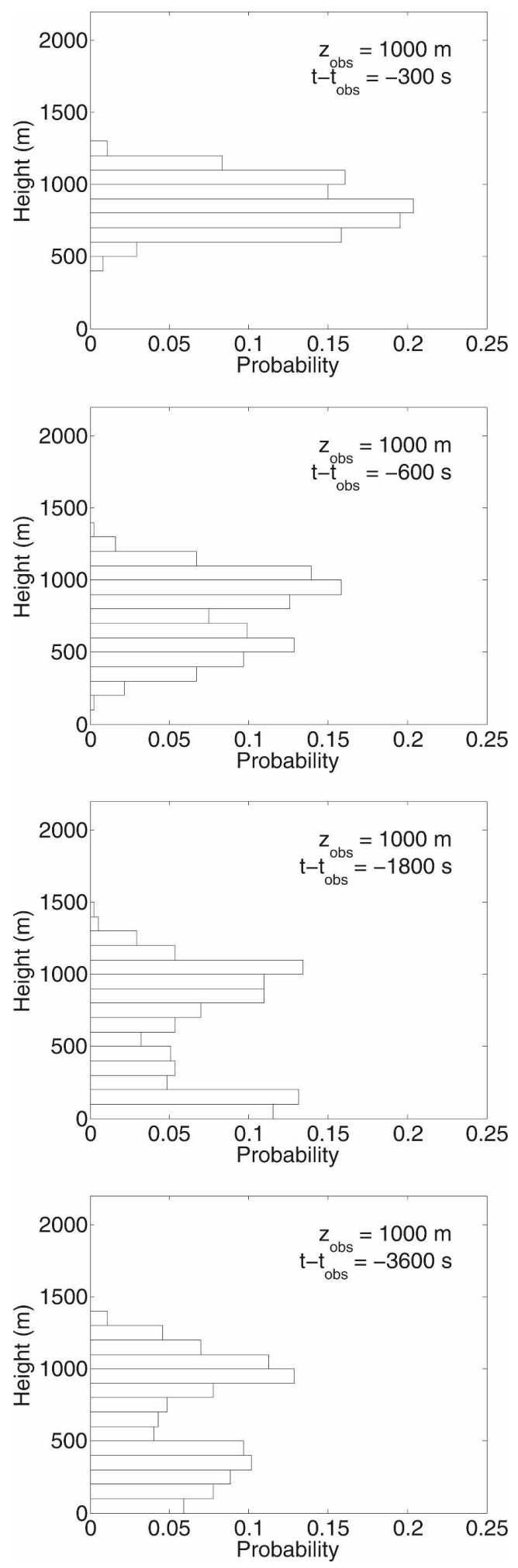

FIG. 6. PDF of the height of the particles evolving in time for $z_{\text {obs }}=1000 \mathrm{~m}$. (top to bottom) Instances where $t^{\prime}=-300, t^{\prime}=$ $-600, t^{\prime}=-1800$, and $t^{\prime}=-3600 \mathrm{~s}$.

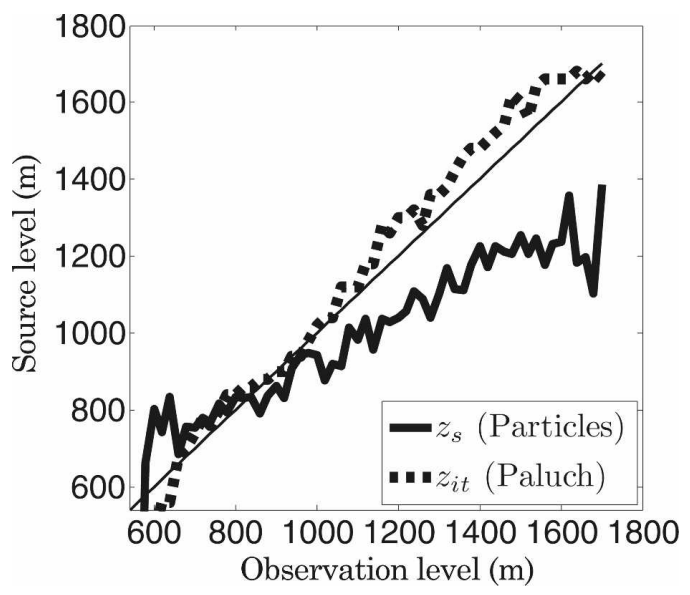

FIG. 7. Source level as a function of observation height, following Paluch analysis (dashed line) and the source-level $z_{s}$ as defined in the text (solid line).

account. This way, biases due to spatial fluctuations in cloud-base height or recirculation from the cloud layer into the subcloud layer, for example, can be eliminated.

Looking at the distribution of cloud-layer particles in Fig. 6, it is clear that hardly anything originates from more than $200 \mathrm{~m}$ above $z_{\text {obs }}$. The cloud influences the environment and particles move toward $z_{\text {obs }}$ only in the 5 min preceding the observation time.

The two source levels $z_{s}$ and $z_{i t}$ can now be plotted against $z_{\text {obs }}$ (Fig. 7). For higher observation levels, a clear discrepancy can here be observed between the source-level $z_{i t}$ (dashed line) inferred from the Paluch analysis and the origin $z_{s}$ of the particles (solid line).

\section{Ensemble averaging over the cloud field}

The previous section focused on the inspection of a single cloud, which is useful for comparisons between this study and earlier observations. However, the rich dataset of LES can now be used to obtain source heights averaged over a very large number of clouds. This reduces the influence of stochastic events and advances the results toward a more statistical representation of the cloud field. The difference between this approach and a bulk parameterization should be stressed: the aim here is to understand the physical properties of the individual clouds by careful conditional sampling and rescaling of the clouds, not to represent the entire field as a (not necessarily physically realistic) bulk cloud. As a consequence, any cloud-top mixing should still be visible in the results.

Taking a representative average over the entire ensemble of clouds has to be done with some caution. For instance, averaging over an ensemble of clouds of dif- 
TABLE 2. Summary of the key variables used in section 4.

\begin{tabular}{ll}
\hline \multicolumn{1}{c}{ Variable } & \multicolumn{1}{c}{ Description } \\
\hline$t_{\text {obs }}$ & Moment of observation \\
$t_{\text {in }}$ & Moment the particle enters the cloud \\
$t_{\text {out }}$ & Moment the particle leaves the cloud \\
$h(t)$ & Cloud height \\
$h_{\text {max }}$ & max $\left[h\left(t_{\text {in }}: t_{\mathrm{obs}}\right)\right]$; maximum height of the cloud between entry time and observation \\
$z^{\prime}(t)$ & $\frac{z(t)-z_{\mathrm{cb}}}{h_{\max }} ;$ relative height of the particle with respect to the cloud \\
& $\frac{z\left(t_{\text {in }}\right)-z_{\mathrm{cb}}}{h_{\text {in }}} ;$ relative height of the particle at entry time \\
$z_{\text {in }}^{\prime}$ & $\frac{z\left(t_{\text {out }}\right)-z_{\mathrm{cb}}}{h_{\text {max }}} ;$ relative height of the particle when leaving the cloud \\
& Average height of a set of particles \\
$z_{\text {out }}^{\prime}$ & Average height of all particles with $z>\bar{z}$ \\
$\bar{z}$ & Average height of all particles with $z<\bar{z}$ \\
$\bar{z}^{+}$ & Particle height at $t=t_{\text {in }}-1800 \mathrm{~s}$ \\
$\bar{z}^{-}$ & Particle height at $t=t_{\text {in }}$ \\
Source level & Particle height at $t=t_{\mathrm{obs}}$ \\
Entry level & Particle height at $t=t_{\text {out }}$ \\
Observation level & Particle height at $t=t_{\text {out }}+1800 \mathrm{~s}$ \\
Exit level &
\end{tabular}

ferent sizes requires an accurate definition of the cloud size; the dynamics of a small chunk broken from a cloud can be expected to differ from a large cloud topping a thermal. Another point is that turbulent diffusion at the cloud edge might cause many particles entering and immediately leaving a cloud without causing significant mixing.

To take these effects into account, results are split between ensembles over all particles that reach the cloud and particles that reach the cloud core (i.e., $\theta_{v}>$ $\left.\theta_{v \text {,env }}\right)$ at some point during their residence in the cloud. To remove passive clouds and dissipating chunks from the ensemble, only clouds with a height of at least 300 $\mathrm{m}$ are taken into account. To see whether clouds reaching the inversion layer have different properties, a distinction is made between all clouds larger than $300 \mathrm{~m}$ and clouds larger than $1000 \mathrm{~m}$.

A subtle point is the exact definition of cloud height for normalizing the height where a particle enters or leaves a cloud. This height $h(t)$, defined as the height difference between the highest and the lowest levels of adjacent gridpoints where $q_{l}>0$, can vary strongly in time between the moment when a parcel enters the cloud $t_{\text {in, }}$ the moment of observation within the cloud $t_{\mathrm{obs}}$, and the moment when the parcel leaves the cloud $t_{\text {out }}$. As a guideline, the maximum height $h_{\max } \equiv$ $\max \left[h\left(t_{\mathrm{in}}: t_{\mathrm{obs}}\right)\right]$ is taken of the cloud between the time of entry $t_{\text {in }}$ and the moment of interest, yielding a normalized height $z^{\prime}(t)=\left[z(t)-z_{\mathrm{cb}}\right] / h_{\max }$, where $z_{\mathrm{cb}}$ is the height of the cloud base. The justification of this choice is that the history of the cloud is relevant for the position of a particle, but the future of the cloud is not. The relative entry level is thus defined as $z_{\text {in }}^{\prime}=\left[z\left(t_{\mathrm{in}}\right)-z_{\mathrm{cb}}\right] / h_{\mathrm{in}}$, and the relative exit level as $z_{\text {out }}^{\prime}=\left[z\left(t_{\text {out }}\right)-z_{\mathrm{cb}}\right] / h_{\text {max }}$. With these definitions, cloud-top entrainment results in a high value for the relative entry-level $z^{\prime}$ in, even in the case of an ascending cloud. This definition also results in a relative exit level that is unbiased by chunks breaking from the main cloud: if, for instance, a chunk breaks from the cloud at midlevel and then dissipates, thus leaving the particle in the environment, $z_{\text {out }}^{\prime}$ would be equal to 0.5 , which is reasonable looking from the perspective of the main cloud. For quick reference, an overview of the definitions used in this section is given in Table 2.

In Fig. 8, the fraction of particles entering or leaving at a relative height $z^{\prime}$ is shown for all four sampling conditions: 1) clouds larger than $300 \mathrm{~m}$; 2) clouds larger than $1000 \mathrm{~m}$; 3) particles reaching the positively buoyant core of clouds that are larger than $300 \mathrm{~m}$; 4) particles reaching the core of clouds larger than $1000 \mathrm{~m}$. The overall look of the results is quite similar for all conditions: the inflow peaks sharply at cloud base, there exists a region between $z^{\prime}=0.2$ and $z^{\prime}=0.6$ where the inflow approximately balances the outflow, and the cloud-environment interaction is constant in height. In the top part of the cloud, inflow decreases, but outflow does not. Because $z_{\text {in }}^{\prime}$ is scaled with the total cloud height at that moment, this strongly suggests an absence of cloud-top mixing. Note that the rescaling of clouds also eliminates effects of reduction of cloud fraction with height; only the geometry of individual clouds (in the sense of a conelike shape, e.g.) could still have an influence. 



FIG. 8. Relative number of particles entering (dashed line) or leaving (dotted line) the cloud as a function of the relative height $h$ for clouds of at least (left) 300 or (right) $1000 \mathrm{~m}$. The top graphs include all events of entering/ leaving a cloud; the results in the bottom figures are conditionally sampled over particles that reach the cloud core (i.e., are positively buoyant at some time between entering and leaving the cloud).

Although Fig. 8 shows that entrainment and detrainment are balanced at midcloud, there are also regions of net in- or outflow. Most pronounced is the large outlet of air in the cloud-top region for the largest clouds, which can be associated with the intrusion in the inversion layer and a resulting increased (anvil-like) outflow. Another interesting difference is the net exchange rate in the midlayer of the cloud: the small clouds display more particles leaving the cloud than entering it, whereas the largest clouds, especially their cores, are net-entraining air from the environment. This is consistent with Neggers et al. (2003) who found a mass flux increasing with height for large clouds.

The equivalent of Fig. 7 (i.e., observation level versus entrance level) in the field-averaged approach can now give more conclusive evidence on the role of lateral and cloud-top entrainment. As is illustrated schematically in Fig. 9, cloud-top entrainment will result in emphasis on the top horizontal bar. Because the definition of $z^{\prime}$ accounts for ascending cloud tops, any signal at or below the diagonal must be due to lateral mixing, but for the lower horizontal bar, which signifies the inflow from the subcloud layer. As can be seen in Fig. 10, no cloud-top mixing is observed anywhere in the cloud field. Aside from an expected strong inflow at cloud base, lateral entrainment is clearly dominating cloud mixing. For smaller clouds, some air can be seen to come from higher levels but not specifically from cloud top. Moreover, this band of in-cloud downdrafts is relatively much smaller for large clouds and can best be explained by the in-cloud turbulence. The complementary graph of Fig. 10 - the relative vertical position where air leaves the cloud as a function of observation level-is shown in Fig. 11. Here, it can be seen that most detrainment occurs at or slightly above observation level. As in Fig. 8, a relatively large outlet of air is observed around $z_{\text {out }}^{\prime}=0.8$, especially for the cloud core of large clouds. Rather unsurprisingly, hardly any particle observed in the cloud core leaves the cloud at a lower level.

The role of the cloud in the dynamics of the cloud layer can be felt not only within the cloud itself but also 


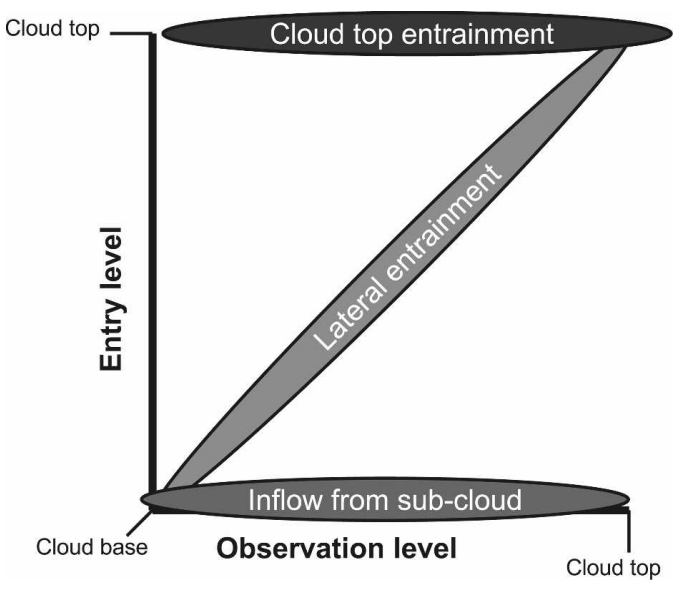

FIG. 9. Conceptual picture of an observation- vs source-level diagram. Inflow from the subcloud layer will show up at the base of the graph, and cloud-top entrainment will appear at the top; the diagonal signifies lateral entrainment. in its immediate surroundings. To illustrate this, the average height $\bar{z}$ of particles entering large clouds at $1200 \mathrm{~m}$ is shown in Fig. 12 as a function of time before and after the moment of entry $t=t_{\text {in }}$. The same is done in Fig. 13 for particles leaving the cloud at $1200 \mathrm{~m}$ with reference to exit time $t=t_{\mathrm{out}}$. While some air is indeed coming from above cloud top and descends $500 \mathrm{~m}$ in the $250 \mathrm{~s}$ before entering, these downdrafts are extremely rare; half an hour before entering, the average height $\bar{z}$ of the particles is $1050 \mathrm{~m}$ and the average height $\bar{z}^{+}$of all particles above $\bar{z}$ is $1200 \mathrm{~m}$, that is, equal to entrance height.

For the outflow the picture is somewhat different: a small portion of air continues to rise (possibly after reentraining into the cloud), but the average height clearly decreases after the detrainment event with 0.2 $\mathrm{m} \mathrm{s}^{-1}$ in the first $500 \mathrm{~s}$. That the detrainment happens far below cloud top suggests that the downward motion
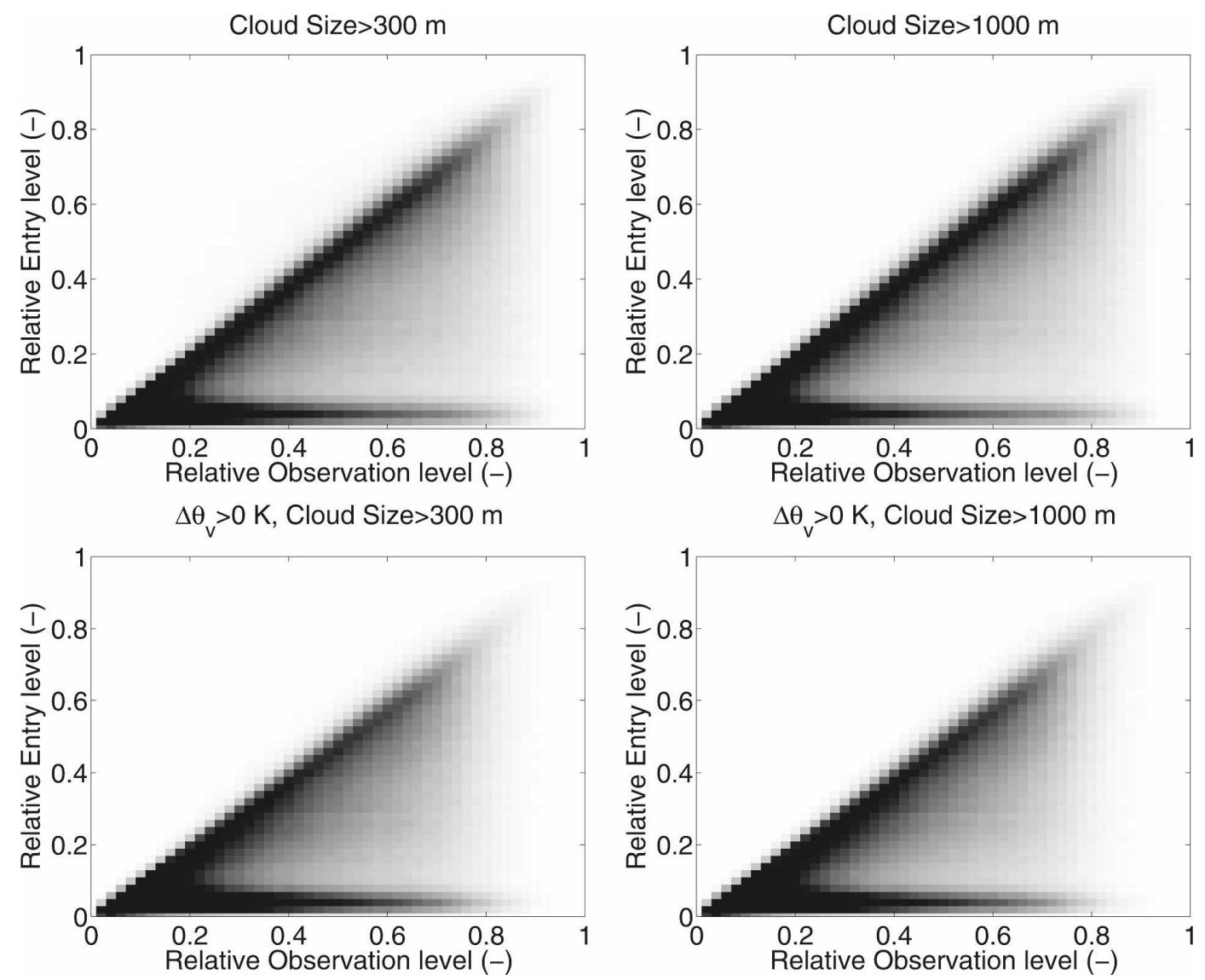

FIG. 10. The relative height at which particles entered the cloud as a function of relative observation level. Distinction is made between clouds with a height of at least (left) 300 or (right) $1000 \mathrm{~m}$. In the bottom panels, the particles are furthermore conditionally sampled on their presence in the cloud core; that is, they had to be positively buoyant at some time between entering and leaving the cloud. 

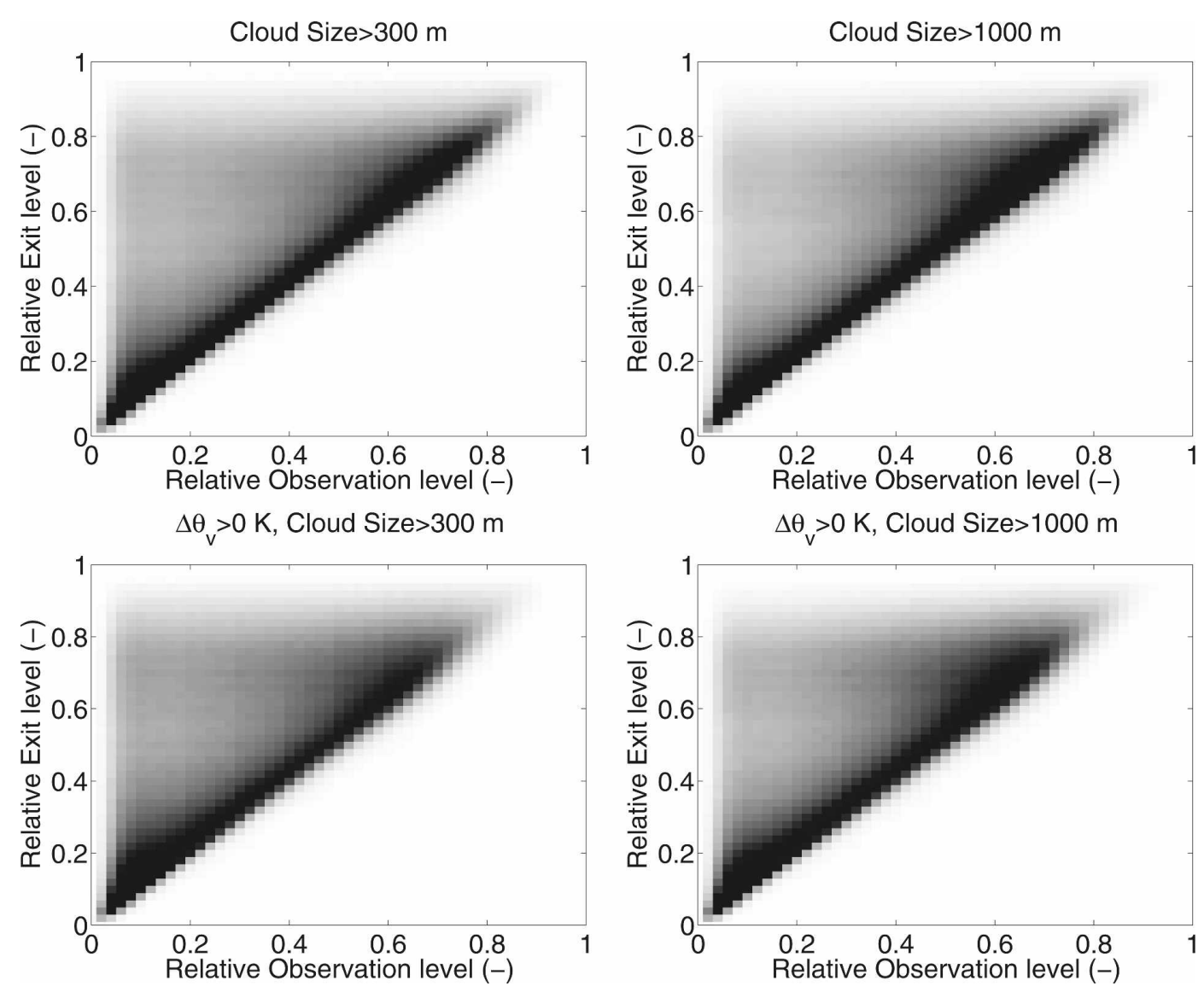

FIG. 11. The relative height at which particles leave the cloud as a function of relative observation level. Distinction is made between clouds with a height of at least (left) 300 or (right) $1000 \mathrm{~m}$ and between the (top) entire cloud and the (bottom) cloud core.

can be associated with the descending shell (see Heus and Jonker 2007; Jonker et al. 2008).

\section{Net vertical transport due to clouds}

As discussed in section 4 , the influence of clouds on the vertical transport of air is not limited to flow within the cloud itself. To give an overall view of the vertical transport due to the cloud, three stages are distinguished in the residence of a particle in the (near vicinity of the) cloud: 1) the $30 \mathrm{~min}$ before entering the cloud, 2) the time between entering and leaving the cloud, and 3) the $30 \mathrm{~min}$ after having left the cloud. This is done by defining, apart from the entry level and the exit level, the source level as the height of a parcel of air 30 min before having entered the cloud and the destination level as the height of a parcel of air $30 \mathrm{~min}$ after having left the cloud. As shown in section 3, this halfhour time window is long enough to include all interactions of the particle with the cloud while excluding interaction with later clouds.
The behavior of air before entering the cloud, during residence in the cloud and after having left the cloud, is shown (from top to bottom) in Fig. 14. Because the tallest clouds have nearly identical cloud tops between 1600 and $1800 \mathrm{~m}$, their heights can be shown in absolute numbers, allowing for inclusion of the subcloud layer in the discussion. Looking at Fig. 14a, most air clearly enters the cloud at cloud base coming from the subcloud layer as expected; air that enters the cloud laterally mostly originates from entry-level height. No clear evidence of descending air (be it by the recirculation vortex or the subsiding shell) can be found in this figure. A minor fraction of the entrained air originates from other (mainly lower) levels; this is probably air that has left and then immediately reentered the cloud. Once air has entered the cloud (depicted in Fig. 14b), it is likely to leave the cloud again at a level equal to or higher than entry level. A cloud core consisting of subcloud-layer air is clearly visible in the form of a dark vertical band at an entry level of $550 \mathrm{~m}$. Penetrative in-cloud downdrafts would be seen in the lower- 


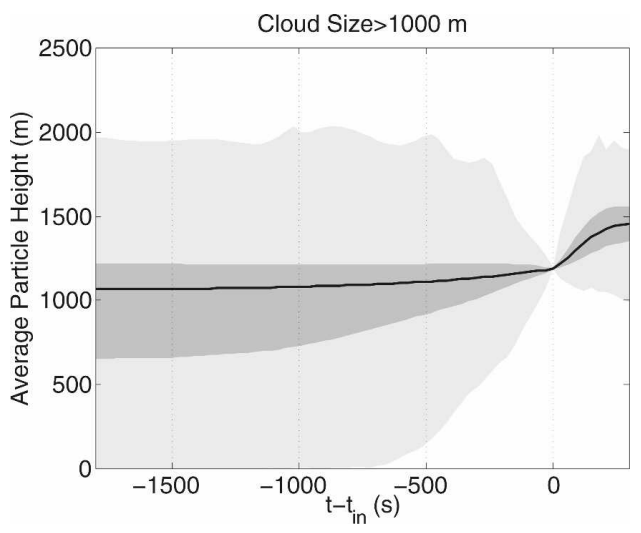

FIG. 12. Average height as a function of time of all particles entering kilometer-sized clouds at $1200 \mathrm{~m}$. For $t<t_{\text {in }}$, particles are located in the environment; for $t>t_{\mathrm{in}}$, particles are located within the cloud. The line denotes the average height $\bar{z}$ of all particles. The edges between the dark and light gray areas are the average of all particles below $\bar{z}$, indicated in the text by $\bar{z}^{-}$, and particles above $\bar{z}$, indicated by $\bar{z}^{+}$. The light gray area envelops the entire particle distribution.

right triangle of the figure, but they are not observed here.

In contrast with the time before entering the cloud, the fingerprint of the subsiding shell is clearly visible for air that has left the cloud as the dark area below the diagonal in Fig. 14c. The appearance of this dark area for all exit levels suggests that this downdraft is indeed due to the subsiding shell, not to the recirculation vortex that would mark only the area around cloud top. The cloud-top region around $1500 \mathrm{~m}$ shows only a somewhat larger downdraft population, while the particle descent remains around $200 \mathrm{~m}$, similar to particles exiting lower in the cloud. It is interesting to note that as a result, in the lowest part of the cloud layer, the subsiding shell enhances mixing of cloud-layer air into the subcloud layer.

The total net vertical transport by the cloud and its surroundings is the resultant of the three graphs in Fig. 14, shown in Fig. 15, where the destination level is plotted against the source level. Clearly, it can be seen that the downward motion of air having left the cloud is strong enough to result in a net downward displacement for a significant number of air parcels. The fact that this downward transport is equally strong at all heights shows that the role of cloud-top-driven downflow is subordinate in the dynamics of shallow cumulus clouds.

\section{Conclusions}

In this paper, the origins of in-cloud air have been studied for shallow cumulus clouds in LES by two dif-

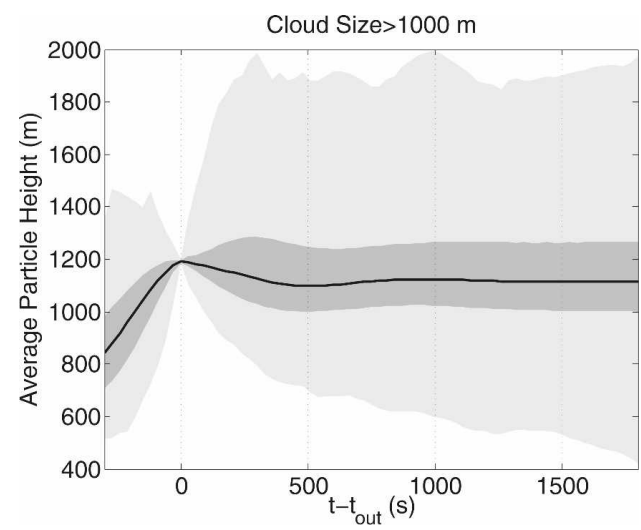

FIG. 13. Average height as a function of time of all particles having left the kilometer-sized clouds at $1200 \mathrm{~m}$. For $t<t_{\text {out }}$, particles are located in the cloud; for $t>t_{\text {out }}$, particles are located within the environment. See Fig. 12 for further explanation.

ferent methods: Paluch diagrams and Lagrangian tracer particles. From the Paluch diagrams, an inferred source level around observation height was obtained, which is also often found in the literature. However, the source level found by explicit backtracking was significantly lower than observation level. In the analysis of individual clouds as well as in ensemble averaging of the entire cloud field, no evidence could be found for significant cloud-top mixing.

Why the Paluch diagrams overpredict the source level and why they correlate so well with two-point mixing remains not entirely clear. Most of the inherent correlation between the pair of conserved variables has been eliminated by the use of the optically optimized $\theta_{\alpha}$ temperature scale. The use of $\theta_{\alpha}$ also widened the allowed regions of buoyancy sorting from TB91, which suggests that buoyancy sorting alone cannot explain the linear distribution of points in the Paluch diagram. One possible explanation might be found in the pathlines within the diagram of diluting parcels in a laterally entraining cloud; lateral entrainment tends to pull already-diluted air toward the line between the properties of less-diluted air and the properties of observation level. Another reason for the overprediction of the source level might be that the inferred source level is calculated in phase space, thus giving too much weight to air parcels with a large deviation from the mean temperature or moisture-most prominently, air coming from above the inversion.

The conceptual picture of a cloud emerging from this study is schematically shown in Fig. 16. A cloud core consisting of air originating from the subcloud layer is recognizable throughout the entire cloud and is constantly diluted by lateral mixing. Part of the laterally entrained air is lifted to higher levels, but another part 




(a)

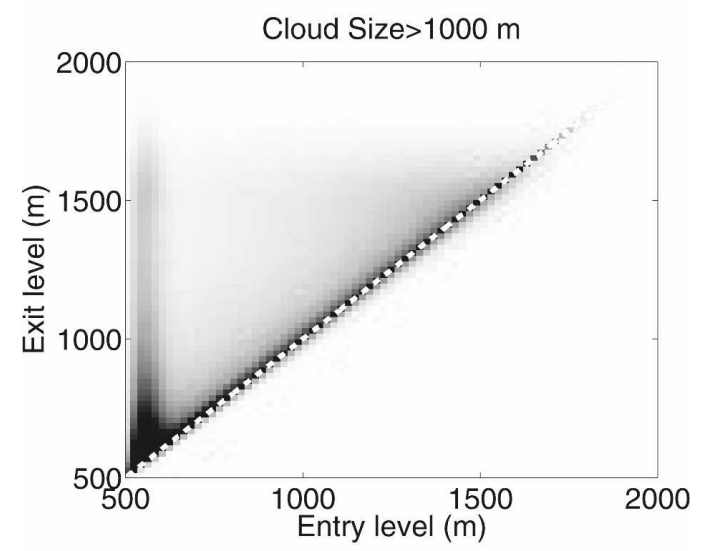

(b)

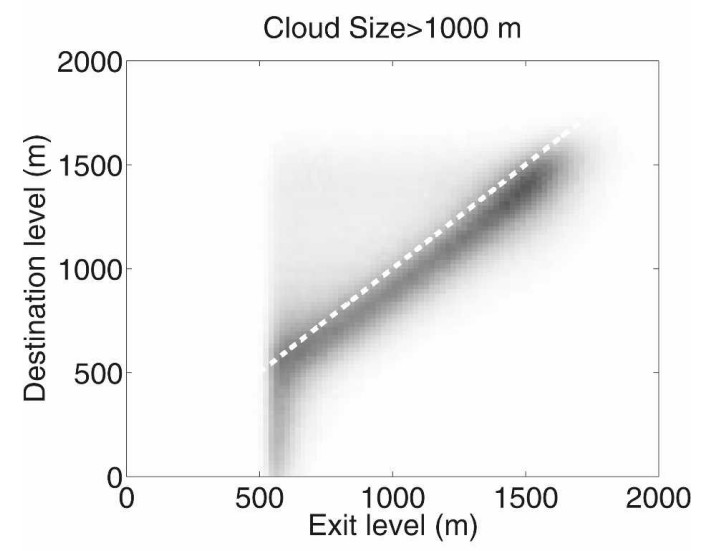

(c)

FIG. 14. The effect of the three stages on the cloud-environment interaction for clouds larger than $1000 \mathrm{~m}$. (a) Source level vs entry level, (b) exit level vs entry level, and (c) destination level vs exit level.

leaves the cloud at levels comparable with the source level. This is in agreement with the results of Kuang and Bretherton (2006), for example, who found hardly any undiluted parcels above cloud base. Also, their ob-

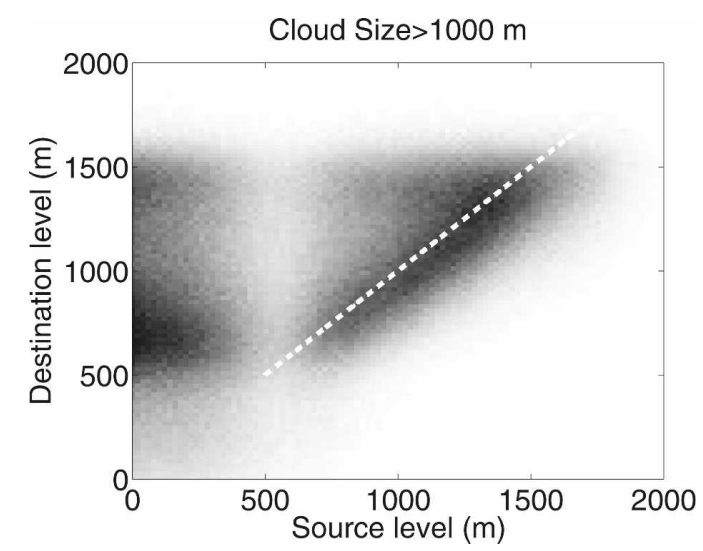

FIG. 15. The destination level vs the source level for clouds larger than $1000 \mathrm{~m}$.

served similarity between deep and shallow cumuli is supported by the similarity in Fig. 4 between our Paluch diagrams and the ones found for much deeper cumuli by Blyth et al. (1988). The specific scaling of the entrainment height showed no evidence in favor of entrainment due to the ascending cloud top, as discussed in Blyth (1993) and Zhao and Austin (2005b), but it should be noted that life cycle effects such as pulsating growth of cumuli (e.g., as discussed in French et al. 1999; Zhao and Austin 2005b) have not been taken into account here and demand further investigation. The subsidence due to evaporative cooling after detrainment from the cloud ensures that some of this air is net transported downward during its stay in or near the cloud. It is notable that air entrained into the cloud appears to be less affected by the subsiding shell than the air detrained from the cloud. This is consistent with the results of Heus and Jonker (2008) and Jonker et al.

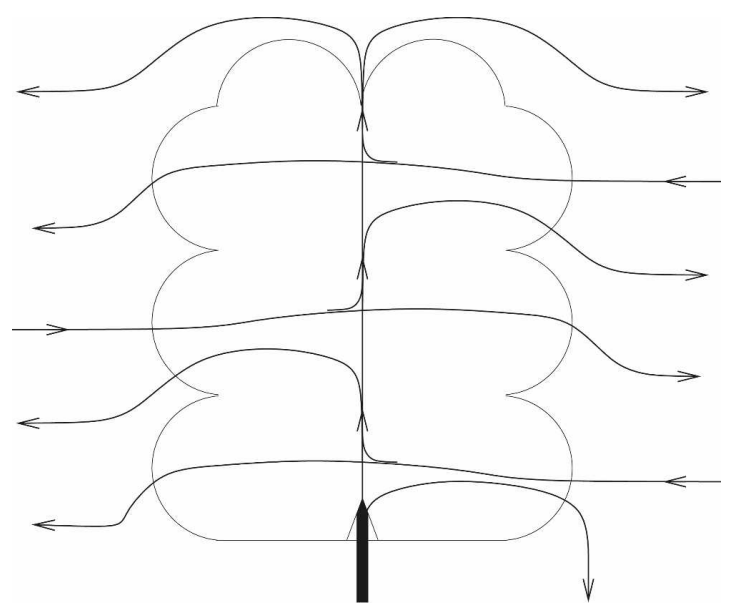

FIG. 16. A conceptual picture of cumulus cloud mixing resulting from this study. 


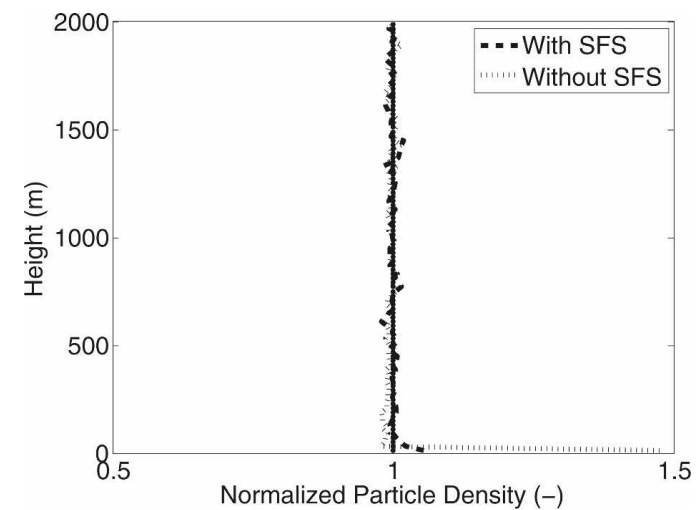

FIG. A1. The normalized particle density at $t=7 \mathrm{~h}$ as function of height for a simulation with (dashed line) and without (dotted line) SFS diffusion.

(2008), who found a shell induced by evaporation of detrained air parcels, even though environmental air can only be transported downward if dragged along with the descending shell.

Acknowledgments. The authors thank Stephan de Roode (KNMI) for his comments and views. The investigations were supported by the Netherlands Organization for Scientific Research (NWO). This work was sponsored by the National Computing Facilities Foundation (NCF) for the use of supercomputer facilities.

\section{APPENDIX}

\section{Validation of Tracer Particles in Cumulus Flow}

The used LPDM has been tested in the clear CBL (Weil et al. 2004) but not in cumulus-topped boundary layers. We define two criteria for validation of the LPDM: 1) a homogeneous particle distribution remains homogeneous in incompressible flow and 2) Lagrangian and Eulerian statistics must converge for a sufficient number of particles.

\section{a. Homogeneity of the distribution}

The particle distribution at $t=7 \mathrm{~h}$ (i.e., $4 \mathrm{~h}$ after initialization of the particles) has been investigated with regard to homogeneity of the particle distribution. The initial focus is on the particle density as a function of height, which is normalized by the total number of particles divided by the number of bins, meaning that the expected value of this function is 1 for all heights. In Fig. A1, it can be seen that for a large part of the domain, this expectation is met both for particles advected with (dashed line) and without SFS diffusion (dotted line). However, the amount of particles in the

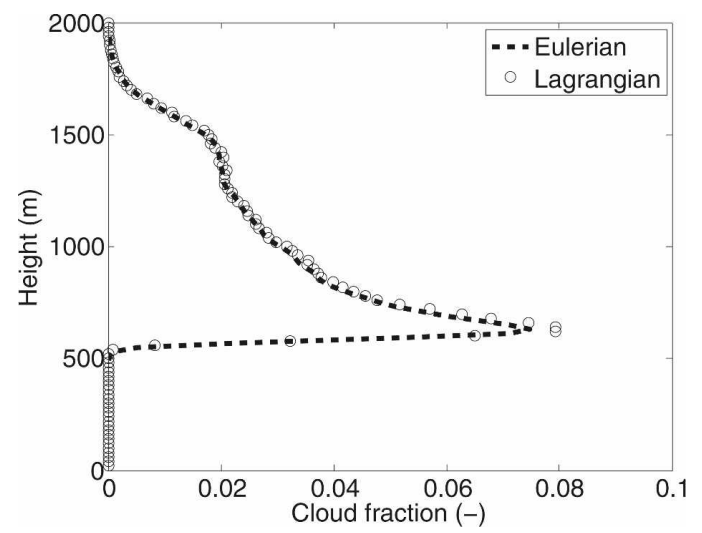

Fig. A2. The cloud fraction between $t=6 \mathrm{~h}, 50 \mathrm{~min}$, and $t=7$ $\mathrm{h}$ as a function of height according to Eulerian (dashed line) and Lagrangian (circles) statistics.

surface layer exceeds the average by $50 \%$ when the SFS scheme is turned off. This is enough to drain a significant amount of particles from the subcloud layer. This behavior could be expected because in the surface layer, the influence of subgrid processes is much more significant than in the bulk of the domain. With the particle SFS scheme turned on, the excess is reduced to a few percent only. Because this effect is rather small and the field of interest of this study lies far away from the surface, it is deemed to be insignificant for this study; it should, however, be taken into consideration in studies of the surface layer.

The homogeneity of the distribution can also be validated by comparing the cloud fraction observed from both the Eulerian and the Lagrangian viewpoints. This is of interest not only because clouds are the topic of interest of this study but also because clouds are most likely to be the subject of inhomogeneities, as they represent the most turbulent structures of the domain. The cloud fraction as a function of height (Fig. A2) shows a general agreement between Lagrangian (circles) and Eulerian (dashed line) statistics, but for the cloud fraction at cloud base, where the peak in cloud fraction is undersampled in Eulerian statistics.

\section{b. Comparison between Eulerian and Lagrangian statistics}

If the massless particles are both well distributed and follow the flow correctly, averages of thermodynamical quantities as seen from a Lagrangian point of view should be comparable with the averages taken from the Eulerian viewpoint. In particular, the particle velocity is of interest here: this is calculated more or less autonomously from the LES velocity, but these two must be identical if the particles do follow the flow. 

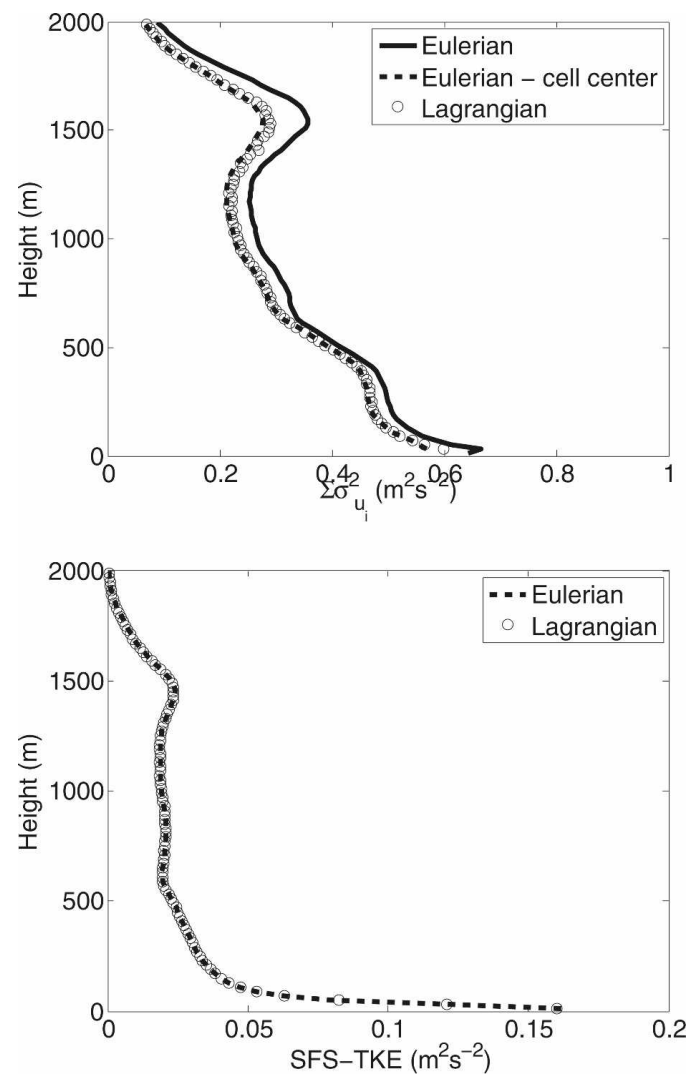

FIG. A3. The slab-averaged sum of variance of the (top) resolved velocities and the (bottom) subfilter-scale TKE between $t=6 \mathrm{~h}, 50 \mathrm{~min}$, and $t=7 \mathrm{~h}$ as function of height according to Eulerian (solid line) and Lagrangian (circles) statistics. For the resolved variance, the dashed line denotes Eulerian statistics after interpolation of the velocities to the cell center.

In Fig. A3, the mean sum of the variances of the resolved velocities and the SFS-TKE is plotted as a function of height from both the Eulerian and the Lagrangian viewpoints. Although the SFS-TKE agrees

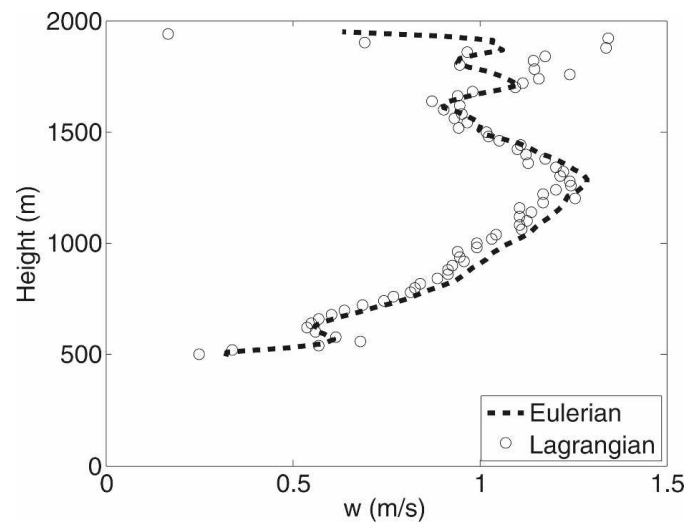

FIG. A4. In-cloud average of $w$ between $t=6 \mathrm{~h}, 50 \mathrm{~min}$, and $t=7 \mathrm{~h}$, using Eulerian (dashed line) or Lagrangian (circles) statistics. very well, the Lagrangian-resolved variance is slightly lower than the Eulerian version. This is due to the calculation of variances using interpolated velocities (as is done for the Lagrangian statistics), which results in decreased variance. If the same approach is taken in the Eulerian statistics (see the dashed line in Fig. A3), the two approaches again agree. Although it is important for the SFS parameterization to calculate the variance correctly, this difference in interpolation does not influence the dynamics of the particles very much, because the dependency on the variance of the SFS contribution in the LPDM is much smaller. This is illustrated with the agreement in SFS-TKE between Eulerian and Lagrangian statistics.

Finally, in Fig. A4, the in-cloud vertical velocity is shown. Here, Lagrangian statistics generally yield a slightly lower value than Eulerian statistics do. This can be explained (similar to the interpretation of Fig. A2) by the fact that the particles better sample the region near the edge of a cloud than the fixed Eulerian grid can.

To summarize, the LPDM appears to meet the criteria of a homogeneous distribution and of reliable statistics very well within the bulk of the domain and can be used with confidence for the purposes of this article.

\section{REFERENCES}

Betts, A. K., 1982: Saturation point analysis of moist convective overturning. J. Atmos. Sci., 39, 1484-1505.

Blyth, A. M., 1993: Entrainment in cumulus clouds. J. Appl. Meteor., 32, 626-641.

— W. A. Cooper, and J. B. Jensen, 1988: A study of the source of entrained air in Montana cumuli. J. Atmos. Sci., 45, 3944 3964.

Carpenter, R. L., K. K. Droegemeier, and A. M. Blyth, 1998: Entrainment and detrainment in numerically simulated cumulus congestus clouds. Part III: Parcel analysis. J. Atmos. Sci., 55, 3440-3455.

Cuijpers, J. W. M., and P. G. Duynkerke, 1993: Large eddy simulations of trade wind cumulus clouds. J. Atmos. Sci., 50, 38943908.

Damiani, R., G. Vali, and S. Haimov, 2006: The structure of thermals in cumulus from airborne dual-Doppler radar observations. J. Atmos. Sci., 63, 1432-1450.

Dosio, A., J. V. G. De Arellano, A. A. M. Holtslag, and P. J. H. Builtjes, 2005: Relating Eulerian and Lagrangian statistics for the turbulent dispersion in the atmospheric convective boundary layer. J. Atmos. Sci., 62, 1175-1191.

French, J. R., G. Vali, and R. D. Kelly, 1999: Evolution of small cumulus clouds in Florida: Observations of pulsating growth. Atmos. Res., 52, 143-165.

Heus, T., and H. J. J. Jonker, 2008: Subsiding shells around shallow cumulus clouds. J. Atmos. Sci., 65, 1003-1018.

Heymsfield, A. J., P. N. Johnson, and J. E. Dye, 1978: Observations of moist adiabatic ascent in northeast Colorado cumulus congestus clouds. J. Atmos. Sci., 35, 1689-1703. 
Holland, J. Z., and E. M. Rasmusson, 1973: Measurement of atmospheric mass, energy, and momentum budgets over a 500kilometer square of tropical ocean. Mon. Wea. Rev., 101, 44 55.

Jensen, J. B., P. H. Austin, M. B. Baker, and A. M. Blyth, 1985: Turbulent mixing, spectral evolution and dynamics in a warm cumulus cloud. J. Atmos. Sci., 42, 173-192.

Jonas, P. R., 1990: Observations of cumulus cloud entrainment. Atmos. Res., 25, 105-127.

Jonker, H. J. J., T. Heus, and P. P. Sullivan, 2008: A refined view of vertical mass transport by cumulus convection. Geophys. Res. Lett., 35, L07810, doi:10.1029/2007GL032606.

Kain, J. S., and J. Fritsch, 1993: Convective parameterization for mesoscale models: The Kain-Fritsch scheme. The Representation of Cumulus Convection in Numerical Models, Meteor. Monogr., No. 46, Amer. Meteor. Soc., 165-170.

Kuang, Z., and C. S. Bretherton, 2006: A mass-flux scheme view of a high-resolution simulation of a transition from shallow to deep cumulus convection. J. Atmos. Sci., 63, 1895-1909.

LaMontagne, R. G., and J. W. Telford, 1983: Cloud top mixing in small cumuli. J. Atmos. Sci., 40, 2148-2156.

Lin, C. C., and A. Arakawa, 1997: The macroscopic entrainment processes of simulated cumulus ensemble. Part II. Testing the entraining-plume model. J. Atmos. Sci., 54, 1044-1053.

Neggers, R. A. J., A. P. Siebesma, and H. J. J. Jonker, 2002: A multiparcel model for shallow cumulus convection. J. Atmos. Sci., 59, 1655-1668.

- H. J. J. Jonker, and A. P. Siebesma, 2003: Size statistics of cumulus cloud populations in large-eddy simulations. $J$. Atmos. Sci., 60, 1060-1074.

Paluch, I. R., 1979: The entrainment mechanism in Colorado cumuli. J. Atmos. Sci., 36, 2467-2478.

Raymond, D. J., and M. H. Wilkening, 1982: Flow and mixing in New Mexico mountain cumuli. J. Atmos. Sci., 39, 2211-2228.

Reuter, G. W., and M. K. Yau, 1987a: Mixing mechanisms in cumulus congestus clouds. Part I: Observations. J. Atmos. Sci., 44, 781-797.

$\ldots$, and $\_$1987b: Mixing mechanisms in cumulus congestus clouds. Part II: Numerical simulations. J. Atmos. Sci., 44, 798827.

Rodts, S. M. A., P. G. Duynkerke, and H. J. J. Jonker, 2003: Size distributions and dynamical properties of shallow cumulus clouds from aircraft observations and satellite data. J. Atmos. Sci., 60, 1895-1912.

Siebesma, A. P., and J. W. M. Cuijpers, 1995: Evaluation of parametric assumptions for shallow cumulus convection. J. Atmos. Sci., 52, 650-666.

_ and H. J. J. Jonker, 2000: Anomalous scaling of cumulus cloud boundaries. Phys. Rev. Lett., 85, 214-217.

— , and Coauthors, 2003: A large eddy simulation intercomparison study of shallow cumulus convection. J. Atmos. Sci., 60, 1201-1219.

Squires, P., 1958: The microstructure and colloidal stability of warm clouds. Part I. The relation between structure and stability. Tellus, 10, 256-261.

Stommel, H., 1947: Entrainment of air into a cumulus cloud. $J$. Meteor., 4, 91-94.

Taylor, G. R., and M. B. Baker, 1991: Entrainment and detrainment in cumulus clouds. J. Atmos. Sci., 48, 112-121.

Telford, J. W., and P. B. Wagner, 1974: Measurement of horizontal air motion near clouds from aircraft. J. Atmos. Sci., 31, 2066-2080.

Thomson, D. J., 1987: Criteria for the selection of stochastic models of particle trajectories in turbulent flows. J. Fluid Mech., 180, 529-556.

von Salzen, K., and N. A. McFarlane, 2002: Parameterization of the bulk effects of lateral and cloud-top entrainment in transient shallow cumulus clouds. J. Atmos. Sci., 59, 1405-1430.

Weil, J. C., P. P. Sullivan, and C. H. Moeng, 2004: The use of large-eddy simulations in Lagrangian particle dispersion models. J. Atmos. Sci., 61, 2877-2887.

Zhao, M., and P. H. Austin, 2005a: Life cycle of numerically simulated shallow cumulus clouds. Part I: Transport. J. Atmos. Sci., 62, 1269-1290.

$\longrightarrow$, and $-2005 \mathrm{~b}$ : Life cycle of numerically simulated shallow cumulus clouds. Part II: Mixing dynamics. J. Atmos. Sci., 62, 1291-1310. 